\title{
Oscillations in Difference Equations with Deviating Arguments and Variable Coefficients
}

\author{
G. E. Chatzarakis, ${ }^{1}$ H. Péics, ${ }^{2}$ and I. P. Stavroulakis ${ }^{3}$ \\ ${ }^{1}$ Department of Electrical and Electronic Engineering Educators, School of Pedagogical and Technological Education (ASPETE), \\ 14121 N. Heraklio, Athens, Greece \\ ${ }^{2}$ Faculty of Civil Engineering, University of Novi Sad, 24000 Subotica, Serbia \\ ${ }^{3}$ Department of Mathematics, University of Ioannina, 45110 Ioannina, Greece
}

Correspondence should be addressed to H. Péics; peics@gf.uns.ac.rs

Received 19 May 2014; Accepted 21 July 2014; Published 18 August 2014

Academic Editor: Patricia J. Y. Wong

Copyright ( 2014 G. E. Chatzarakis et al. This is an open access article distributed under the Creative Commons Attribution License, which permits unrestricted use, distribution, and reproduction in any medium, provided the original work is properly cited.

New sufficient conditions for the oscillation of all solutions of difference equations with several deviating arguments and variable coefficients are presented. Examples illustrating the results are also given.

\section{Introduction}

In this paper we study the oscillation of all solutions of difference equation with several variable retarded arguments of the form

$$
\Delta x(n)+\sum_{i=1}^{m} p_{i}(n) x\left(\tau_{i}(n)\right)=0, \quad n \in \mathbb{N}_{0}
$$

and the (dual) difference equation with several variable advanced arguments of the form

$$
\nabla x(n)-\sum_{i=1}^{m} p_{i}(n) x\left(\sigma_{i}(n)\right)=0, \quad n \in \mathbb{N},
$$

where $m \in \mathbb{N},\left(p_{i}(n)\right), 1 \leq i \leq m$, are sequences of positive real numbers and $\left(\tau_{i}(n)\right), 1 \leq i \leq m$, are sequences of integers such that

$$
\begin{gathered}
\tau_{i}(n) \leq n-1, \quad \forall n \in \mathbb{N}_{0}, \\
\lim _{n \rightarrow \infty} \tau_{i}(n)=\infty, \quad 1 \leq i \leq m
\end{gathered}
$$

and $\left(\sigma_{i}(n)\right), 1 \leq i \leq m$, are sequences of integers such that

$$
\sigma_{i}(n) \geq n+1, \quad \forall n \in \mathbb{N}, 1 \leq i \leq m .
$$

Here, $\Delta$ denotes the forward difference operator $\Delta x(n)=$ $x(n+1)-x(n)$ and $\nabla$ denotes the backward difference operator $\nabla x(n)=x(n)-x(n-1)$.

Strong interest in $\left(E_{R}\right)$ is motivated by the fact that it represents a discrete analogue of the differential equation (see $[1-3]$ and the references cited therein)

$$
x^{\prime}(t)+\sum_{i=1}^{m} p_{i}(t) x\left(\tau_{i}(t)\right)=0, \quad t \geq 0,
$$

where, for every $i \in\{1, \ldots, m\}, p_{i}$ is a continuous real-valued function in the interval $[0, \infty)$ and $\tau_{i}$ is a continuous realvalued function on $[0, \infty)$ such that

$$
\tau_{i}(t) \leq t, \quad t \geq 0, \quad \lim _{t \rightarrow \infty} \tau_{i}(t)=\infty,
$$

while $\left(\mathrm{E}_{\mathrm{A}}\right)$ represents a discrete analogue of the advanced differential equation (see $[1,2]$ and the references cited therein)

$$
x^{\prime}(t)-\sum_{i=1}^{m} p_{i}(t) x\left(\sigma_{i}(t)\right)=0, \quad t \geq 1,
$$

where, for every $i \in\{1, \ldots, m\}, p_{i}$ is a continuous real-valued function in the interval $[1, \infty)$ and $\sigma_{i}$ is a continuous realvalued function on $[1, \infty)$ such that

$$
\sigma_{i}(t) \geq t, \quad t \geq 1 .
$$


By a solution of $\left(\mathrm{E}_{\mathrm{R}}\right)$, we mean a sequence of real numbers $(x(n))_{n \geq-w}$ which satisfies $\left(\mathrm{E}_{\mathrm{R}}\right)$ for all $n \geq 0$. Here

$$
w=-\min _{\substack{n \geq 0 \\ 1 \leq i \leq m}} \tau_{i}(n)
$$

It is clear that, for each choice of real numbers $c_{-w}$, $c_{-w+1}, \ldots, c_{-1}, c_{0}$, there exists a unique solution $(x(n))_{n \geq-w}$ of $\left(\mathrm{E}_{\mathrm{R}}\right)$ which satisfies the initial conditions $x(-w)=c_{-w}$, $x(-w+1)=c_{-w+1}, \ldots, x(-1)=c_{-1}, x(0)=c_{0}$.

By a solution of $\left(\mathrm{E}_{\mathrm{A}}\right)$, we mean a sequence of real numbers $(x(n))_{n \geq 0}$ which satisfies $\left(\mathrm{E}_{\mathrm{A}}\right)$ for all $n \geq 1$.

A solution $(x(n))_{n \geq-w}\left(\right.$ or $\left.(x(n))_{n \geq 0}\right)$ of $\left(\mathrm{E}_{\mathrm{R}}\right)\left(\right.$ or $\left.\left(\mathrm{E}_{\mathrm{A}}\right)\right)$ is called oscillatory, if the terms $x(n)$ of the sequence are neither eventually positive nor eventually negative. Otherwise, the solution is said to be nonoscillatory.

In the last few decades, the oscillatory behavior of the solutions of difference and differential equations with several deviating arguments and variable coefficients has been studied. See, for example, [1-14] and the references cited therein.

In 2006, Berezansky and Braverman [5] proved that if

$$
\limsup _{n \rightarrow \infty} \sum_{i=1}^{m} p_{i}(n)>0, \quad \liminf _{n \rightarrow \infty} \sum_{i=1}^{m} \sum_{j=\tau(n)}^{n-1} p_{i}(j)>\frac{1}{e}
$$

where $\tau(n)=\max _{1 \leq i \leq m} \tau_{i}(n)$, for all $n \geq 0$, then all solutions of $\left(E_{R}\right)$ oscillate.

Recently, Chatzarakis et al. [7-9] established the following theorems.

Theorem 1 (see [9]). Assume that the sequences $\left(\tau_{i}(n)\right)$ $\left[\left(\sigma_{i}(n)\right)\right], 1 \leq i \leq m$, are increasing, (1) [(2)] holds, and

$$
\limsup _{n \rightarrow \infty} \sum_{i=1}^{m} \sum_{j=\tau(n)}^{n} p_{i}(j)\left[\limsup _{n \rightarrow \infty} \sum_{i=1}^{m} \sum_{j=n}^{\sigma(n)} p_{i}(j)\right]>1,
$$

where $\tau(n)=\max _{1 \leq i \leq m} \tau_{i}(n)$, for all $n \geq 0,[\sigma(n)=$ $\min _{1 \leq i \leq m} \sigma_{i}(n)$, for all $\left.n \geq 1\right]$, or

$$
\begin{gathered}
\limsup _{n \rightarrow \infty} \sum_{i=1}^{m} p_{i}(n)>0, \\
\liminf _{n \rightarrow \infty} \sum_{i=1}^{m} \sum_{j=\tau_{i}(n)}^{n-1} p_{i}(j)\left[\liminf _{n \rightarrow \infty} \sum_{i=1}^{m} \sum_{j=n+1}^{\sigma_{i}(n)} p_{i}(j)\right]>\frac{1}{e},
\end{gathered}
$$

then all solutions of $\left(E_{R}\right)\left[\left(E_{A}\right)\right]$ oscillate.

Theorem 2 (see $[7,8]$ ). Assume that the sequences $\left(\tau_{i}(n)\right)\left[\left(\sigma_{i}(n)\right)\right], 1 \leq i \leq m$, are increasing and (1) [(2)] holds. Set

$$
\begin{gathered}
\alpha=\min \left\{\alpha_{i}: 1 \leq i \leq m\right\} \\
\alpha_{i}=\liminf _{n \rightarrow \infty} \sum_{j=\tau_{i}(n)}^{n-1} p_{i}(j)\left[\liminf _{n \rightarrow \infty} \sum_{j=n+1}^{\sigma_{i}(n)} p_{i}(j)\right] .
\end{gathered}
$$

If $0<\alpha \leq 1 / e$, and

$$
\begin{aligned}
& \limsup _{n \rightarrow \infty} \sum_{i=1}^{m} \sum_{j=\tau(n)}^{n} p_{i}(j)\left[\limsup _{n \rightarrow \infty} \sum_{i=1}^{m} \sum_{j=n}^{\sigma(n)} p_{i}(j)\right] \\
& >1-(1-\sqrt{1-\alpha})^{2}
\end{aligned}
$$

or

$$
\begin{gathered}
\limsup _{n \rightarrow \infty} \sum_{i=1}^{m} \sum_{j=\tau(n)}^{n} p_{i}(j)\left[\limsup _{n \rightarrow \infty} \sum_{i=1}^{m} \sum_{j=n}^{\sigma(n)} p_{i}(j)\right] \\
>1-\frac{1-\alpha-\sqrt{1-2 \alpha-\alpha^{2}}}{2}
\end{gathered}
$$

then all solutions of $\left(E_{R}\right)\left[\left(E_{A}\right)\right]$ oscillate.

The authors study further $\left(\mathrm{E}_{\mathrm{R}}\right)$ and $\left(\mathrm{E}_{\mathrm{A}}\right)$ and derive new sufficient oscillation conditions. These conditions are the improved and generalized discrete analogues of the oscillation conditions for the corresponding differential equations, which were studied in 1982 by Ladas and Stavroulakis [2]. Examples illustrating the results are also given.

\section{Oscillation Criteria}

2.1. Retarded Difference Equations. We present new sufficient conditions for the oscillation of all solutions of $\left(E_{R}\right)$.

Theorem 3. Assume that $\left(\tau_{i}(n)\right), 1 \leq i \leq m$, are increasing sequences of integers such that (1) holds and $\left(p_{i}(n)\right), 1 \leq i \leq m$, are sequences of positive real numbers and define $\alpha_{i}, 1 \leq i \leq m$, by (11). If $\alpha_{i}>0,1 \leq i \leq m$, and

$$
\left[\prod_{i=1}^{m}\left(\alpha_{i}+\sum_{j=1, j \neq i}^{m} \liminf _{n \rightarrow \infty} \sum_{k=\tau_{j}(n)}^{n-1} p_{i}(k)\right)\right]^{1 / m}>\frac{1}{e},
$$

then all solutions of $\left(E_{R}\right)$ oscillate.

Proof. Assume, for the sake of contradiction, that $(x(n))_{n \geq-w}$ is a nonoscillatory solution of $\left(\mathrm{E}_{\mathrm{R}}\right)$. Then it is either eventually positive or eventually negative. As $(-x(n))_{n>-w}$ is also a solution of $\left(\mathrm{E}_{\mathrm{R}}\right)$, we may restrict ourselves only to the case where $x(n)>0$ for all large $n$. Let $n_{1} \geq-w$ be an integer such that $x(n)>0$ for all $n \geq n_{1}$. Then, there exists $n_{2} \geq n_{1}$ such that

$$
x\left(\tau_{i}(n)\right)>0, \quad \forall n \geq n_{2}, 1 \leq i \leq m .
$$

In view of this, $\left(\mathrm{E}_{\mathrm{R}}\right)$ becomes

$$
\Delta x(n)=-\sum_{i=1}^{m} p_{i}(n) x\left(\tau_{i}(n)\right) \leq 0, \quad \forall n \geq n_{2},
$$

which means that the sequence $(x(n))$ is eventually decreasing.

Next choose a natural number $n_{3}>n_{2}$ such that

$$
x(n)<x\left(\tau_{i}(n)\right), \quad \forall n \geq n_{3}, 1 \leq i \leq m .
$$


Set

$$
\begin{gathered}
z_{i}(n)=\frac{x\left(\tau_{i}(n)\right)}{x(n)}, \quad \forall n \geq n_{3}, 1 \leq i \leq m, \\
\varphi_{i}=\liminf _{n \rightarrow \infty} z_{i}(n), \quad 1 \leq i \leq m .
\end{gathered}
$$

It is obvious that

$$
z_{i}(n)>1, \quad \varphi_{i} \geq 1 \quad \text { for } i=1,2, \ldots, m .
$$

Now we will show that $\varphi_{i}<\infty$ for $i=1,2, \ldots, m$. Indeed, assume that $\varphi_{i}=\infty$ for some $i, i=1,2, \ldots, m$. For this $i$, by $\left(\mathrm{E}_{\mathrm{R}}\right)$, we have

$$
\Delta x(n)+p_{i}(n) x\left(\tau_{i}(n)\right) \leq 0, \quad \forall n \geq n_{3} .
$$

At this point, we will establish the following claim.

Claim 1 (cf. [8]). For each $n \geq n_{3}$, there exists an integer $n_{i}^{*} \geq n$ for each $i=1,2, \ldots, m$ such that $\tau_{i}\left(n_{i}^{*}\right) \leq n-1$, and

$$
\begin{aligned}
& \sum_{j=n}^{n_{i}^{*}} p_{i}(j) \geq \frac{\alpha_{i}-\varepsilon}{2}, \\
& \sum_{j=\tau_{i}\left(n_{i}^{*}\right)}^{n-1} p_{i}(j)>\frac{\alpha_{i}-\varepsilon}{2},
\end{aligned}
$$

where $\varepsilon$ is an arbitrary real number with $0<\varepsilon<\alpha_{i}$.

To prove this claim, let us consider an arbitrary real number $\varepsilon$ with $0<\varepsilon<\alpha_{i}$. Then by (11) we can choose an integer $n_{3} \geq n_{2}$ such that

$$
\sum_{j=\tau_{i}(n)}^{n-1} p_{i}(j) \geq \alpha_{i}-\varepsilon, \quad \forall n \geq n_{3}, \quad 1 \leq i \leq m .
$$

Assume, first, that $p_{i}(n) \geq\left(\alpha_{i}-\varepsilon\right) / 2$ and choose $n_{i}^{*}=n$. Then $\tau_{i}\left(n_{i}^{*}\right)=\tau_{i}(n) \leq n-1$. Moreover, we have

$$
\sum_{j=n}^{n_{i}^{*}} p_{i}(j)=\sum_{j=n}^{n} p_{i}(j)=p_{i}(n) \geq \frac{\alpha_{i}-\varepsilon}{2}
$$

and, by (23),

$$
\sum_{j=\tau_{i}\left(n_{i}^{*}\right)}^{n-1} p_{i}(j)=\sum_{j=\tau_{i}(n)}^{n-1} p_{i}(j)>\frac{\alpha_{i}-\varepsilon}{2} .
$$

So, (21) and (22) are fulfilled. Next, we suppose that $p_{i}(n)<$ $\left(\alpha_{i}-\varepsilon\right) / 2$. It is not difficult to see that (23) guarantees that $\sum_{j=0}^{\infty} p_{i}(j)=\infty$. In particular, it holds

$$
\sum_{j=n}^{\infty} p_{i}(j)=\infty
$$

Thus, as $p_{i}(n)<\left(\alpha_{i}-\varepsilon\right) / 2$, there always exists an integer $n_{i}^{*}>$ $n$ so that

$$
\sum_{j=n}^{n_{i}^{*}-1} p_{i}(j)<\frac{\alpha_{i}-\varepsilon}{2}
$$

and (21) holds. We assert that $\tau_{i}\left(n_{i}^{*}\right) \leq n-1$. Otherwise, $\tau_{i}\left(n_{i}^{*}\right) \geq n$. We also have $\tau_{i}\left(n_{i}^{*}\right) \leq n_{i}^{*}-1$. Hence, in view of (27), we get

$$
\sum_{j=\tau_{i}\left(n_{i}^{*}\right)}^{n_{i}^{*}-1} p_{i}(j) \leq \sum_{j=n}^{n_{i}^{*}-1} p_{i}(j)<\frac{\alpha_{i}-\varepsilon}{2} .
$$

On the other hand, (23) gives

$$
\sum_{j=\tau_{i}\left(n_{i}^{*}\right)}^{n_{i}^{*}-1} p_{i}(j) \geq \alpha_{i}-\varepsilon>\frac{\alpha_{i}-\varepsilon}{2} .
$$

We have arrived at a contradiction, which shows our assertion that $\tau_{i}\left(n_{i}^{*}\right) \leq n-1$. Furthermore, by using (23) (for the integer $\left.n_{i}^{*}\right)$ as well as (27), we obtain

$$
\begin{aligned}
\sum_{j=\tau_{i}\left(n_{i}^{*}\right)}^{n-1} p_{i}(j)= & \sum_{j=\tau_{i}\left(n_{i}^{*}\right)}^{n_{i}^{*}-1} p_{i}(j) \\
& -\sum_{j=n}^{n_{i}^{*}-1} p_{i}(j)>\left(\alpha_{i}-\varepsilon\right)-\frac{\alpha_{i}-\varepsilon}{2} \\
= & \frac{\alpha_{i}-\varepsilon}{2}
\end{aligned}
$$

and consequently (22) holds true. Our claim has been proved.

Now, summing up (20) from $n$ to $n_{i}^{*}$, we find

$$
x\left(n_{i}^{*}+1\right)-x(n)+\sum_{j=n}^{n_{i}^{*}} p_{i}(j) x\left(\tau_{i}(j)\right) \leq 0, \quad \forall n \geq n_{3},
$$

or

$$
\begin{array}{r}
x(n) \geq\left(\sum_{j=n}^{n_{i}^{*}} p_{i}(j)\right) x\left(\tau_{i}\left(n_{i}^{*}\right)\right) \geq \frac{\alpha_{i}-\varepsilon}{2} x\left(\tau_{i}\left(n_{i}^{*}\right)\right), \\
\forall n \geq n_{3} .
\end{array}
$$

Summing up (20) from $\tau_{i}\left(n_{i}^{*}\right)$ to $n-1$, we find

$$
x(n)-x\left(\tau_{i}\left(n_{i}^{*}\right)\right)+\sum_{j=\tau_{i}\left(n_{i}^{*}\right)}^{n-1} p_{i}(j) x\left(\tau_{i}(j)\right) \leq 0,
$$

$$
\forall n \geq n_{3}
$$

or

$$
\begin{aligned}
x\left(\tau_{i}\left(n_{i}^{*}\right)\right) & \geq\left(\sum_{j=\tau_{i}\left(n_{i}^{*}\right)}^{n-1} p_{i}(j)\right) x\left(\tau_{i}(n-1)\right) \\
& \geq \frac{\alpha_{i}-\varepsilon}{2} x\left(\tau_{i}(n)\right), \quad \forall n \geq n_{3} .
\end{aligned}
$$

Combining (32) and (34), we obtain

$$
x(n) \geq\left(\frac{\alpha_{i}-\varepsilon}{2}\right)^{2} x\left(\tau_{i}(n)\right), \quad \forall n \geq n_{3},
$$


or

$$
\frac{x\left(\tau_{i}(n)\right)}{x(n)} \leq\left(\frac{2}{\alpha_{i}-\varepsilon}\right)^{2}, \quad \forall n \geq n_{3}
$$

which means that $\left(z_{i}(n)\right)$ is bounded. This contradicts our assumption that $\varphi_{i}=\infty$. Therefore $\varphi_{i}<\infty$ for every $i=$ $1,2, \ldots, m$.

Dividing both sides of $\left(\mathrm{E}_{\mathrm{R}}\right)$ by $x(n)$, for $n \geq n_{3}$, we obtain

$$
\frac{\Delta x(n)}{x(n)}+\sum_{i=1}^{m} p_{i}(n) \frac{x\left(\tau_{i}(n)\right)}{x(n)}=0
$$

or

$$
\frac{\Delta x(n)}{x(n)}+\sum_{i=1}^{m} p_{i}(n) z_{i}(n)=0
$$

Summing up (38) from $\tau_{\rho}(n)$ to $n-1$ for $\rho=1,2, \ldots, m$, we find

$$
\sum_{j=\tau_{\rho}(n)}^{n-1} \frac{\Delta x(j)}{x(j)}+\sum_{i=1}^{m} \sum_{j=\tau_{\rho}(n)}^{n-1} p_{i}(j) z_{i}(j)=0
$$

But

$$
\begin{aligned}
\sum_{j=\tau_{\rho}(n)}^{n-1} \frac{\Delta x(j)}{x(j)} & =\sum_{j=\tau_{\rho}(n)}^{n-1}\left(\frac{x(j+1)}{x(j)}-1\right) \\
& \geq \sum_{j=\tau_{\rho}(n)}^{n-1}\left(1+\ln \frac{x(j+1)}{x(j)}-1\right) \\
& =\ln \frac{x(n)}{x\left(\tau_{\rho}(n)\right)},
\end{aligned}
$$

or

$$
\sum_{j=\tau_{\rho}(n)}^{n-1} \frac{\Delta x(j)}{x(j)} \geq-\ln z_{\rho}(n), \quad \rho=1,2, \ldots, m .
$$

Combining (39) and (41), we obtain

$$
-\ln z_{\rho}(n)+\sum_{i=1}^{m} \sum_{j=\tau_{\rho}(n)}^{n-1} p_{i}(j) z_{i}(j) \leq 0, \quad \rho=1,2, \ldots, m,
$$

or

$$
\ln z_{\rho}(n) \geq \sum_{i=1}^{m} \sum_{j=\tau_{\rho}(n)}^{n-1} p_{i}(j) z_{i}(j), \quad \rho=1,2, \ldots, m .
$$

Taking limit inferiors on both sides of the above inequalities (43), we obtain

$$
\ln \varphi_{\rho} \geq \sum_{i=1}^{m} \varphi_{i}\left(\liminf _{n \rightarrow \infty} \sum_{k=\tau_{\rho}(n)}^{n-1} p_{i}(k)\right), \quad \rho=1,2, \ldots, m
$$

and by adding we find

$$
\sum_{i=1}^{m} \ln \varphi_{i} \geq \sum_{i=1}^{m} \varphi_{i}\left(\sum_{j=1}^{m} \liminf _{n \rightarrow \infty} \sum_{k=\tau_{j}(n)}^{n-1} p_{i}(k)\right) .
$$

Set

$$
\begin{aligned}
& f\left(\varphi_{1}, \varphi_{2}, \ldots, \varphi_{m}\right) \\
& \quad \equiv \sum_{i=1}^{m} \ln \varphi_{i}-\sum_{i=1}^{m} \varphi_{i}\left(\sum_{j=1}^{m} \liminf _{n \rightarrow \infty} \sum_{k=\tau_{j}(n)}^{n-1} p_{i}(k)\right) .
\end{aligned}
$$

Clearly

$$
f\left(\varphi_{1}, \varphi_{2}, \ldots, \varphi_{m}\right) \geq 0, \quad \forall \varphi_{1}, \varphi_{2}, \ldots, \varphi_{m} \geq 1 .
$$

Since

$$
\frac{\partial f}{\partial \varphi_{i}}=\frac{1}{\varphi_{i}}-\sum_{j=1}^{m} \liminf _{n \rightarrow \infty} \sum_{k=\tau_{j}(n)}^{n-1} p_{i}(k)=0
$$

for

$$
\varphi_{i}=\frac{1}{\sum_{j=1}^{m} \liminf _{n \rightarrow \infty} \sum_{k=\tau_{j}(n)}^{n-1} p_{i}(k)}, \quad i=1,2, \ldots, m,
$$

the function $f$ has a maximum at the critical point

$$
\begin{aligned}
& \left(\frac{1}{\sum_{j=1}^{m} \liminf _{n \rightarrow \infty} \sum_{k=\tau_{j}(n)}^{n-1} p_{1}(k)},\right. \\
& \left.\ldots, \frac{1}{\sum_{j=1}^{m} \liminf _{n \rightarrow \infty} \sum_{k=\tau_{j}(n)}^{n-1} p_{m}(k)}\right)
\end{aligned}
$$

since the quadratic form

$$
\sum_{i, j=1}^{m} \frac{\partial^{2} f}{\partial \varphi_{i} \partial \varphi_{j}} b_{i} b_{j}=-\sum_{i=1}^{m} \frac{b_{i}^{2}}{\varphi_{i}^{2}}<0 .
$$

Since $f\left(\varphi_{1}, \varphi_{2}, \ldots, \varphi_{m}\right) \geq 0$, the maximum of $f$ at the critical point should be nonnegative. Thus,

$$
\sum_{i=1}^{m}\left[-\ln \left(\sum_{j=1}^{m} \liminf _{n \rightarrow \infty} \sum_{k=\tau_{j}(n)}^{n-1} p_{i}(k)\right)\right]-m \geq 0,
$$

that is,

$$
\begin{aligned}
\max _{\varphi_{i} \geq 1} f\left(\varphi_{1}, \varphi_{2}, \ldots, \varphi_{m}\right) \\
=-\ln \prod_{i=1}^{m}\left(\sum_{j=1}^{m} \liminf _{n \rightarrow \infty} \sum_{k=\tau_{j}(n)}^{n-1} p_{i}(k)\right)-m .
\end{aligned}
$$

Hence

$$
\prod_{i=1}^{m}\left(\sum_{j=1}^{m} \liminf _{n \rightarrow \infty} \sum_{k=\tau j(n)}^{n-1} p_{i}(k)\right) \leq \frac{1}{e^{m}}
$$


or

$$
\left[\prod_{i=1}^{m}\left(\alpha_{i}+\sum_{j=1, j \neq i}^{m} \liminf _{n \rightarrow \infty} \sum_{k=\tau_{j}(n)}^{n-1} p_{i}(k)\right)\right]^{1 / m} \leq \frac{1}{e},
$$

which contradicts (14).

The proof of the theorem is complete.

Theorem 4. Assume that $\left(\tau_{i}(n)\right), 1 \leq i \leq m$, are increasing sequences of integers such that (1) holds and $\left(p_{i}(n)\right), 1 \leq i \leq m$, are sequences of positive real numbers and define $\alpha_{i}, 1 \leq i \leq m$, by (11). If $\alpha_{i}>0,1 \leq i \leq m$, and

$$
\begin{aligned}
& \frac{1}{m} \sum_{i=1}^{m} \alpha_{i}+\frac{2}{m} \sum_{i<j}^{m}\left(\liminf _{n \rightarrow \infty} \sum_{k=\tau_{j}(n)}^{n-1} p_{i}(k)\right. \\
&\left.\quad \times \liminf _{n \rightarrow \infty} \sum_{k=\tau_{i}(n)}^{n-1} p_{j}(k)\right)^{1 / 2}>\frac{1}{e},
\end{aligned}
$$

then all solutions of $\left(E_{R}\right)$ oscillate.

Proof. Assume, for the sake of contradiction, that $(x(n))_{n \geq-w}$ is a nonoscillatory solution of $\left(\mathrm{E}_{\mathrm{R}}\right)$. Then it is either eventually positive or eventually negative. As $(-x(n))_{n>-w}$ is also a solution of $\left(\mathrm{E}_{\mathrm{R}}\right)$, we may restrict ourselves only to the case where $x(n)>0$ for all large $n$. Let $n_{1} \geq-w$ be an integer such that $x(n)>0$ for all $n \geq n_{1}$. Then, there exists $n_{2} \geq n_{1}$ such that

$$
x\left(\tau_{i}(n)\right)>0, \quad \forall n \geq n_{2}, \quad 1 \leq i \leq m .
$$

In view of this, $\left(\mathrm{E}_{\mathrm{R}}\right)$ becomes

$$
\Delta x(n)=-\sum_{i=1}^{m} p_{i}(n) x\left(\tau_{i}(n)\right) \leq 0, \quad \forall n \geq n_{2},
$$

which means that the sequence $(x(n))$ is eventually decreasing.

Taking into account the fact that $\varphi_{i}<\infty$ for $i=$ $1,2, \ldots, m$ (see proof of Theorem 3 ), by using (44) and the fact that

$$
\frac{1}{e}>\frac{\ln \varphi_{\rho}}{\varphi_{\rho}}, \quad \rho=1,2, \ldots, m
$$

we obtain

$$
\frac{1}{e}>\sum_{i=1}^{m}\left(\liminf _{n \rightarrow \infty} \sum_{k=\tau_{j}(n)}^{n-1} p_{i}(k)\right) \frac{\varphi_{i}}{\varphi_{j}}, \quad j=1,2, \ldots, m .
$$

Adding these inequalities we have

$$
\begin{aligned}
\frac{m}{e} \geq \sum_{i=1}^{m}\left(\liminf _{n \rightarrow \infty} \sum_{k=\tau_{i}(n)}^{n-1} p_{i}(k)\right) \\
+\sum_{\substack{i<j \\
i, j=1}}^{m}\left[\left(\liminf _{n \rightarrow \infty} \sum_{k=\tau_{j}(n)}^{n-1} p_{i}(k)\right) \frac{\varphi_{i}}{\varphi_{j}}\right. \\
\left.+\left(\liminf _{n \rightarrow \infty} \sum_{k=\tau_{i}(n)}^{n-1} p_{j}(k)\right) \frac{\varphi_{j}}{\varphi_{i}}\right]
\end{aligned}
$$

$$
\begin{aligned}
\geq \sum_{i=1}^{m}\left(\liminf _{n \rightarrow \infty} \sum_{k=\tau_{i}(n)}^{n-1} p_{i}(k)\right) \\
+2 \sum_{\substack{i<j \\
i, j=1}}^{m}\left[\left(\liminf _{n \rightarrow \infty} \sum_{k=\tau_{j}(n)}^{n-1} p_{i}(k)\right)\right. \\
\left.\times\left(\liminf _{n \rightarrow \infty} \sum_{k=\tau_{i}(n)}^{n-1} p_{j}(k)\right)\right]^{1 / 2},
\end{aligned}
$$

or

$$
\begin{aligned}
\frac{1}{m} \sum_{i=1}^{m} \alpha_{i}+\frac{2}{m} \sum_{i<j}^{m} & \left(\liminf _{n \rightarrow \infty} \sum_{k=1}^{n-1} p_{i}(k)\right. \\
& \left.\times \liminf _{n \rightarrow \infty} \sum_{k=\tau_{i}(n)}^{n-1} p_{j}(k)\right)^{1 / 2} \leq \frac{1}{e},
\end{aligned}
$$

which contradicts (56).

The proof of the theorem is complete.

2.2. Advanced Difference Equations. Similar oscillation theorems for the (dual) advanced difference equation $\left(\mathrm{E}_{\mathrm{A}}\right)$ can be derived easily. The proofs of these theorems are omitted, since they follow a similar procedure as in Section 2.1.

Theorem 5. Assume that $\left(\sigma_{i}(n)\right), 1 \leq i \leq m$, are increasing sequences of integers such that (2) holds and $\left(p_{i}(n)\right), 1 \leq i \leq m$, are sequences of positive real numbers and define $\alpha_{i}, 1 \leq i \leq m$, by (11). If $\alpha_{i}>0,1 \leq i \leq m$, and

$$
\left[\prod_{i=1}^{m}\left(\alpha_{i}+\sum_{j=1, j \neq i}^{m} \liminf _{n \rightarrow \infty} \sum_{k=n+1}^{\sigma_{j}(n)} p_{i}(k)\right)\right]^{1 / m}>\frac{1}{e},
$$

then all solutions of $\left(E_{A}\right)$ oscillate.

Theorem 6. Assume that $\left(\sigma_{i}(n)\right), 1 \leq i \leq m$, are increasing sequences of integers such that (2) holds and $\left(p_{i}(n)\right), 1 \leq i \leq m$, are sequences of positive real numbers and define $\alpha_{i}, 1 \leq i \leq m$, by (11). If $\alpha_{i}>0,1 \leq i \leq m$, and

$$
\begin{aligned}
\frac{1}{m} \sum_{i=1}^{m} \alpha_{i}+\frac{2}{m} \sum_{\substack{i<j \\
i, j=1}}^{m}\left(\liminf _{n \rightarrow \infty} \sum_{k=n+1}^{\sigma_{j}(n)} p_{i}(k)\right. & \\
& \left.\times \liminf _{n \rightarrow \infty} \sum_{k=n+1}^{\sigma_{i}(n)} p_{j}(k)\right)^{1 / 2}>\frac{1}{e},
\end{aligned}
$$

then all solutions of $\left(E_{A}\right)$ oscillate.

2.3. Special Cases. In the case where $p_{i}, i=1,2, \ldots, m$, are positive real constants and $\tau_{i}$ are constant retarded arguments of the form $\tau_{i}(n)=n-k_{i}$, [ $\sigma_{i}$ are constant advanced arguments 
of the form $\left.\sigma_{i}(n)=n+k_{i}\right], k_{i} \in \mathbb{N}, i=1,2, \ldots, m$, equation $\left(\mathrm{E}_{\mathrm{R}}\right)\left[\left(\mathrm{E}_{\mathrm{A}}\right)\right]$ takes the form

$$
\begin{aligned}
& \Delta x(n)+\sum_{i=1}^{m} p_{i} x\left(n-k_{i}\right)=0, \quad n \in \mathbb{N}_{0}, \\
& {\left[\nabla x(n)-\sum_{i=1}^{m} p_{i} x\left(n+k_{i}\right)=0, n \in \mathbb{N}\right] .}
\end{aligned}
$$

For this equation, as a consequence of Theorems 3 [5] and $4[6]$, we have the following corollary.

Corollary 7. Assume that

$$
\left[\prod_{i=1}^{m} p_{i}\right]^{1 / m}\left(\sum_{i=1}^{m} k_{i}\right)>\frac{1}{e},
$$

or

$$
\frac{1}{m}\left(\sum_{i=1}^{m} \sqrt{p_{i} k_{i}}\right)^{2}>\frac{1}{e} .
$$

Then all solutions of $(E)$ oscillate.

Remark 8. A research question that arises is whether Theorems 3-6 are valid, even in the case where the coefficients $p(n)$ oscillate (see $[15,16])$. Then our results would be comparable to those in $[15,16]$. This is a question that we currently study and expect to have some results soon.

\section{Examples}

The following two examples illustrate that the conditions for oscillations (65) and (66) are independent. They are chosen in such a way that only one of them is satisfied.

Example 1. Consider the retarded difference equation

$$
\begin{gathered}
\Delta x(n)+\frac{1}{5 e} x(n-1)+\frac{1}{6 e} x(n-2) \\
+\frac{1000}{7198 e} x(n-3)=0, \quad n \in \mathbb{N}_{0} .
\end{gathered}
$$

Here $m=3, \tau_{1}(n)=n-1, \tau_{2}(n)=n-2, \tau_{3}(n)=n-3$, and

$$
\tau(n)=\max _{1 \leq i \leq 3} \tau_{i}(n)=n-1 .
$$

It is easy to see that

$$
\begin{aligned}
& {\left[\prod_{i=1}^{3} p_{i}\right]^{1 / 3}\left(\sum_{i=1}^{3} k_{i}\right)} \\
& \quad=\sqrt[3]{\frac{1}{5 e} \cdot \frac{1}{6 e} \cdot \frac{1000}{7198 e}} \cdot(1+2+3) \simeq 0.3679135 \\
& \quad>\frac{1}{e} .
\end{aligned}
$$

That is, condition (65) of Corollary 7 is satisfied and therefore all solutions of equation (67) oscillate.
However,

$$
\begin{aligned}
& \frac{1}{3}\left(\sum_{i=1}^{3} \sqrt{p_{i} k_{i}}\right)^{2} \\
& \quad=\frac{1}{3}\left(\sqrt{\frac{1}{5 e} \cdot 1}+\sqrt{\frac{1}{6 e} \cdot 2}+\sqrt{\frac{1000}{7198 e} \cdot 3}\right)^{2} \simeq 0.3420547 \\
& \quad<\frac{1}{e}
\end{aligned}
$$

That is, condition (66) of Corollary 7 is not satisfied.

Observe that

$$
\begin{aligned}
& \alpha_{1}=\liminf _{n \rightarrow \infty} \sum_{j=n-1}^{n-1} p_{1}=\frac{1}{5 e} \simeq 0.0735758, \\
& \alpha_{2}=\liminf _{n \rightarrow \infty} \sum_{j=n-2}^{n-1} p_{2}=\frac{2}{6 e} \simeq 0.1226264, \\
& \alpha_{3}=\liminf _{n \rightarrow \infty} \sum_{j=n-3}^{n-1} p_{3}=\frac{3000}{7198 e} \simeq 0.1533256 .
\end{aligned}
$$

Thus

$$
\alpha=\min \left\{\alpha_{i}: 1 \leq i \leq 3\right\} \simeq 0.0735758<\frac{1}{e} .
$$

Also,

$$
\begin{aligned}
& \limsup _{n \rightarrow \infty} \sum_{i=1}^{3} \sum_{j=n-1}^{n} p_{i} \\
& =\limsup _{n \rightarrow \infty}\left[2 \cdot \frac{1}{5 e}+2 \cdot \frac{1}{6 e}+2 \cdot \frac{1000}{7198 e}\right] \simeq 0.3719953 \\
& <1, \\
& 0.3719953<1-(1-\sqrt{1-\alpha})^{2} \simeq 0.9985944, \\
& 0.3719953<1-\frac{1-\alpha-\sqrt{1-2 \alpha-\alpha^{2}}}{2} \simeq 0.997069, \\
& \liminf _{n \rightarrow \infty} \sum_{i=1}^{3} \sum_{j=n-1}^{n-1} p_{i}=\liminf _{n \rightarrow \infty}\left[\frac{1}{5 e}+\frac{1}{6 e}+\frac{1000}{7198 e}\right] \\
& \simeq 0.1859976<\frac{1}{e}, \\
& \liminf _{n \rightarrow \infty} \sum_{i=1}^{3} \sum_{j=\tau_{i}(n)}^{n-1} p_{i}(j)=\liminf _{n \rightarrow \infty}\left[\frac{1}{5 e}+\frac{2}{6 e}+\frac{3000}{7198 e}\right] \\
& \simeq 0.349528<\frac{1}{e}
\end{aligned}
$$

and therefore none of the conditions (9), (12), (13), (8), and (10) are satisfied. 
Example 2. Consider the advanced difference equation

$$
\nabla x(n)-\frac{5}{100} x(n+1)-\frac{22}{100} x(n+2)=0, \quad n \in \mathbb{N} .
$$

Here $m=2, \sigma_{1}(n)=n+1, \sigma_{2}(n)=n+2$, and

$$
\sigma(n)=\min _{1 \leq i \leq 2} \sigma_{i}(n)=n+1
$$

It is easy to see that

$$
\begin{aligned}
\frac{1}{2}\left(\sum_{i=1}^{2} \sqrt{p_{i} k_{i}}\right)^{2} & =\frac{1}{2}\left(\sqrt{\frac{5}{100} \cdot 1}+\sqrt{\frac{22}{100} \cdot 2}\right)^{2} \\
& \simeq 0.393323969>\frac{1}{e} .
\end{aligned}
$$

That is, condition (66) of Corollary 7 is satisfied and therefore all solutions of (74) oscillate.

However,

$$
\begin{aligned}
{\left[\prod_{i=1}^{2} p_{i}\right]^{1 / 2}\left(\sum_{i=1}^{2} k_{i}\right) } & =\sqrt{\frac{5}{100} \cdot \frac{22}{100}} \cdot(1+2) \\
& \simeq 0.314642654<\frac{1}{e} .
\end{aligned}
$$

That is, condition (65) of Corollary 7 is not satisfied.

Observe that

$$
\begin{gathered}
\alpha_{1}=\liminf _{n \rightarrow \infty} \sum_{j=n+1}^{n+1} p_{1}=\frac{5}{100}=0.05, \\
\alpha_{2}=\liminf _{n \rightarrow \infty} \sum_{j=n+1}^{n+2} p_{2}=2 \cdot \frac{22}{100}=0.44 .
\end{gathered}
$$

Thus

$$
\alpha=\min \left\{\alpha_{i}: 1 \leq i \leq 2\right\}=0.05<\frac{1}{e} .
$$

Also,

$$
\begin{gathered}
\limsup _{n \rightarrow \infty} \sum_{i=1}^{2} \sum_{j=n}^{n+1} p_{i}=\limsup _{n \rightarrow \infty}\left[2 \cdot \frac{5}{100}+2 \cdot \frac{22}{100}\right]=0.54<1, \\
0.54<1-(1-\sqrt{1-\alpha})^{2} \simeq 0.999358869 \\
0.54<1-\frac{1-\alpha-\sqrt{1-2 \alpha-\alpha^{2}}}{2} \simeq 0.998682383
\end{gathered}
$$

and therefore none of the conditions (9), (12), and (13) are satisfied.

At this point, we give an example with general retarded arguments illustrating the main result of Theorem 3. Similarly, one can construct examples to illustrate Theorems 4-6.
Example 3. Consider the delay difference equation

$$
\Delta x(n)+\frac{c}{3 n} x([0.5 n])+\frac{2 c}{3 n} x([0.2 n])=0, \quad n \geq 10,
$$

with $c=17 / 50$.

Here $\tau_{1}(n)=[0.5 n]$ and $\tau_{2}(n)=[0.2 n]$ denote the integer parts of $0.5 n$ and $0.2 n$. Observe that the sequences $\tau_{1}(n)$ and $\tau_{2}(n)$ are increasing, $\lim _{n \rightarrow \infty} \tau_{1}(n)=+\infty, \lim _{n \rightarrow \infty} \tau_{2}(n)=$ $+\infty$, and

$$
\begin{aligned}
\tau_{1}(n)=[0.5 n] \leq 0.5 n<n & \text { or } & \tau_{1}(n) \leq n-1, \\
\tau_{2}(n)=[0.2 n] \leq 0.2 n<n & \text { or } & \tau_{2}(n) \leq n-1, \\
& & \forall n \geq 10 .
\end{aligned}
$$

Observe that, for a positive decreasing function $f(x)$, the following inequality holds:

$$
\int_{b-1}^{b} f(x) d x \geq f(b) \geq \int_{b}^{b+1} f(x) d x
$$

Based on the above inequality, we will show that

$$
\liminf _{n \rightarrow \infty} \sum_{k=[(a / b) n]}^{n-1} \frac{Q}{k}=Q \cdot \ln \frac{b}{a}
$$

for any $a, b \in \mathbb{N}, a<b$, and any real number $Q$. Indeed,

$$
\begin{aligned}
& \sum_{k=[(a / b) n]}^{n-1} \frac{Q}{k} \geq Q \sum_{k=[(a / b) n]}^{n-1} \int_{k}^{k+1} \frac{d s}{s} \\
&=Q \int_{[(a / b) n]}^{n} \frac{d s}{s}=Q \cdot \ln \frac{n}{[(a / b) n]}, \\
& \sum_{k=[(a / b) n]}^{n-1} \frac{Q}{k} \leq Q \sum_{k=[(a / b) n]}^{n-1} \int_{k-1}^{k} \frac{d s}{s} \\
&=Q \int_{[(a / b) n]-1}^{n-1} \frac{d s}{s}=Q \cdot \ln \frac{n-1}{[(a / b) n]-1} .
\end{aligned}
$$

It is easy to see that

$$
\lim _{n \rightarrow \infty} Q \cdot \ln \frac{n}{[(a / b) n]}=\lim _{n \rightarrow \infty} Q \cdot \ln \frac{n-1}{[(a / b) n]-1}=Q \cdot \ln \frac{b}{a} .
$$

From the above, it follows that

$$
\liminf _{n \rightarrow \infty} \sum_{k=[(a / b) n]}^{n-1} \frac{Q}{k}=Q \cdot \ln \frac{b}{a}
$$


Therefore

$$
\begin{gathered}
\alpha_{1}=\liminf _{n \rightarrow \infty} \sum_{k=[0.5 n]}^{n-1} \frac{c}{3 k}=\frac{c}{3} \ln 2>0, \\
\alpha_{2}=\liminf _{n \rightarrow \infty} \sum_{k=[0.2 n]}^{n-1} \frac{2 c}{3 k}=\frac{2 c}{3} \ln 5>0, \\
\liminf _{n \rightarrow \infty} \sum_{k=[0.2 n]}^{n-1} \frac{c}{3 k}=\frac{c}{3} \ln 5, \\
\liminf _{n \rightarrow \infty} \sum_{k=[0.5 n]}^{n-1} \frac{2 c}{3 k}=\frac{2 c}{3} \ln 2 .
\end{gathered}
$$

Hence

$$
\begin{aligned}
& {\left[\prod_{i=1}^{2}\left(\alpha_{i}+\sum_{j=1, j \neq i}^{2} \liminf _{n \rightarrow \infty} \sum_{k=\tau_{j}(n)}^{n-1} p_{i}(k)\right)\right]^{1 / 2}} \\
& \quad=\sqrt{\left[\frac{c}{3} \ln 2+\frac{c}{3} \ln 5\right] \cdot\left[\frac{2 c}{3} \ln 2+\frac{2 c}{3} \ln 5\right]} \\
& \quad=\frac{c \sqrt{2} \ln 10}{3} \simeq 0.369052667>\frac{1}{e} .
\end{aligned}
$$

That is, condition (14) of Theorem 3 is satisfied and therefore all solutions of (81) oscillate.

Observe, however, that

$$
\begin{aligned}
\sum_{i=1}^{2} \sum_{j=\tau(n)}^{n-1} p_{i}(j) & =\sum_{j=[0.5 n]}^{n-1} \frac{c}{3 j}+\sum_{j=[0.5 n]}^{n-1} \frac{2 c}{3 j} \\
& =\sum_{j=[0.5 n]}^{n-1} \frac{c}{j} \longrightarrow c \ln 2 \simeq 0.235670041 \\
\sum_{i=1}^{2} \sum_{j=\tau(n)}^{n} p_{i}(j) & =\sum_{j=[0.5 n]}^{n} \frac{c}{3 j}+\sum_{j=[0.5 n]}^{n} \frac{2 c}{3 j} \\
& =\sum_{j=[0.5 n]}^{n} \frac{c}{j} \longrightarrow c \ln 2 \simeq 0.235670041
\end{aligned}
$$$$
\text { as } n \longrightarrow \infty \text {. }
$$

Thus

$$
\begin{aligned}
& \liminf _{n \rightarrow \infty} \sum_{i=1}^{2} \sum_{j=\tau(n)}^{n-1} p_{i}(j) \\
& \quad=\limsup _{n \rightarrow \infty} \sum_{i=1}^{m} \sum_{j=\tau(n)}^{n} p_{i}(j) \simeq 0.235670041<\frac{1}{e} .
\end{aligned}
$$

Also,

$$
\begin{gathered}
\alpha=\min \left\{\alpha_{i}: 1 \leq i \leq 2\right\}=\alpha_{1}=\frac{c}{3} \ln 2 \simeq 0.07855668, \\
0.235670041<1-(1-\sqrt{1-\alpha})^{2} \simeq 0.998393464, \\
0.235670041<1-\frac{1-\alpha-\sqrt{1-2 \alpha-\alpha^{2}}}{2} \simeq 0.996639107
\end{gathered}
$$

and therefore none of the conditions (8), (9), (12), and (13) are satisfied.

\section{Conflict of Interests}

The authors declare that there is no conflict of interests regarding the publication of this paper.

\section{Acknowledgment}

The second author was supported by the Serbian Ministry of Science, Technology and Development for Scientific Research Grant no. III44006.

\section{References}

[1] N. Fukagai and T. Kusano, "Oscillation theory of first order functional-differential equations with deviating arguments," Annali di Matematica Pura ed Applicata. Serie Quarta, vol. 136, pp. 95-117, 1984.

[2] G. Ladas and I. P. Stavroulakis, "Oscillations caused by several retarded and advanced arguments," Journal of Differential Equations, vol. 44, no. 1, pp. 134-152, 1982.

[3] B. Li, "Oscillation of first order delay differential equations," Proceedings of the American Mathematical Society, vol. 124, no. 12, pp. 3729-3737, 1996.

[4] O. Arino, I. Györi, and A. Jawhari, "Oscillation criteria in delay equations," Journal of Differential Equations, vol. 53, no. 1, pp. $115-123,1984$.

[5] L. Berezansky and E. Braverman, "On existence of positive solutions for linear difference equations with several delays," Advances in Dynamical Systems and Applications, vol. 1, no. 1, pp. 29-47, 2006.

[6] L. Berezansky and E. Braverman, "Positive solutions for a scalar differential equation with several delays," Applied Mathematics Letters, vol. 21, no. 6, pp. 636-640, 2008.

[7] G. E. Chatzarakis, T. Kusano, and I. P. Stavroulakis, "Oscillation conditions for difference equations with several variable," to appear in Mathematica Bohemica.

[8] G. E. Chatzarakis, J. Manojlovic, S. Pinelas, and I. P. Stavroulakis, "Oscillation criteria of difference equations with several deviating arguments," to appear in Yokohama Mathematical Journal.

[9] G. E. Chatzarakis, S. Pinelas, and I. P. Stavroulakis, "Oscillations of difference equations with several deviated arguments," to appear in Aequationes Mathematicae.

[10] M. K. Grammatikopoulos, R. Koplatadze, and I. P. Stavroulakis, "On the oscillation of solutions of first order differential equations with retarded arguments," Georgian Mathematical Journal, vol. 10, no. 1, pp. 63-76, 2003.

[11] B. R. Hunt and J. A. Yorke, "When all solutions of $x^{\prime}=$ $\sum_{j=1}^{n} q_{j}(t) x\left(t-T_{j}(t)\right)$ oscillate," Journal of Differential Equations, vol. 53, no. 2, pp. 139-145, 1984.

[12] J. Jaroš and I. P. Stavroulakis, "Necessary and sufficient conditions for oscillations of difference equations with several delays," Utilitas Mathematica, vol. 45, pp. 187-195, 1994.

[13] X. N. Luo, Y. Zhou, and C. F. Li, "Oscillation of a nonlinear difference equation with several delays," Mathematica Bohemica, vol. 128, no. 3, pp. 309-317, 2003. 
[14] X. H. Tang and R. Y. Zhang, "New oscillation criteria for delay difference equations," Computers \& Mathematics with Applications, vol. 42, no. 10-11, pp. 1319-1330, 2001.

[15] L. Berezansky, G. E. Chatzarakis, A. Domoshnitsky, and I. P. Stavroulakis, "Oscillations of difference equations with several oscillating coefficients," Abstract and Applied Analysis, vol. 2014, Article ID 392097, 9 pages, 2014.

[16] M. Bohner, G. E. Chatzarakis, and I. P. Stavroulakis, "Qualitative behavior of solutions of difference equations with several oscillating coefficients," Arabian Journal of Mathematics, vol. 3, no. 1, pp. 1-13, 2014. 


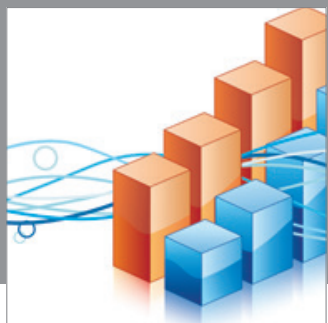

Advances in

Operations Research

mansans

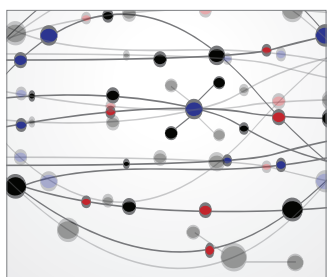

The Scientific World Journal
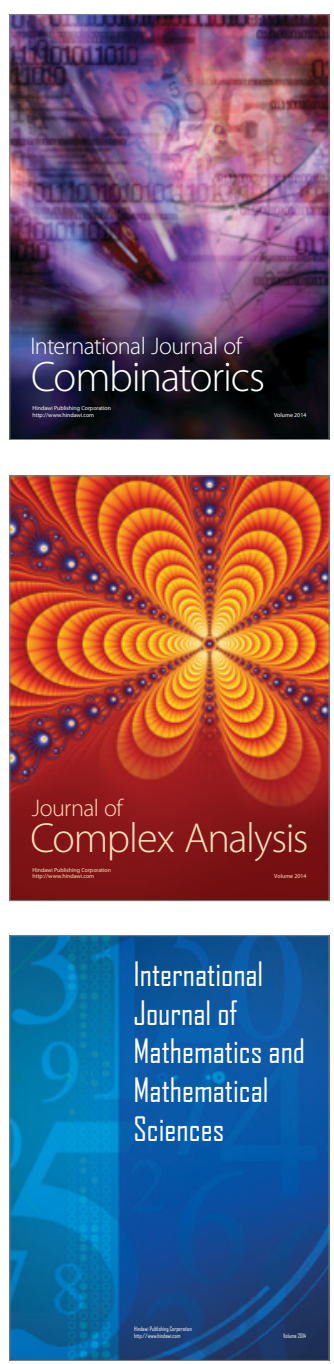
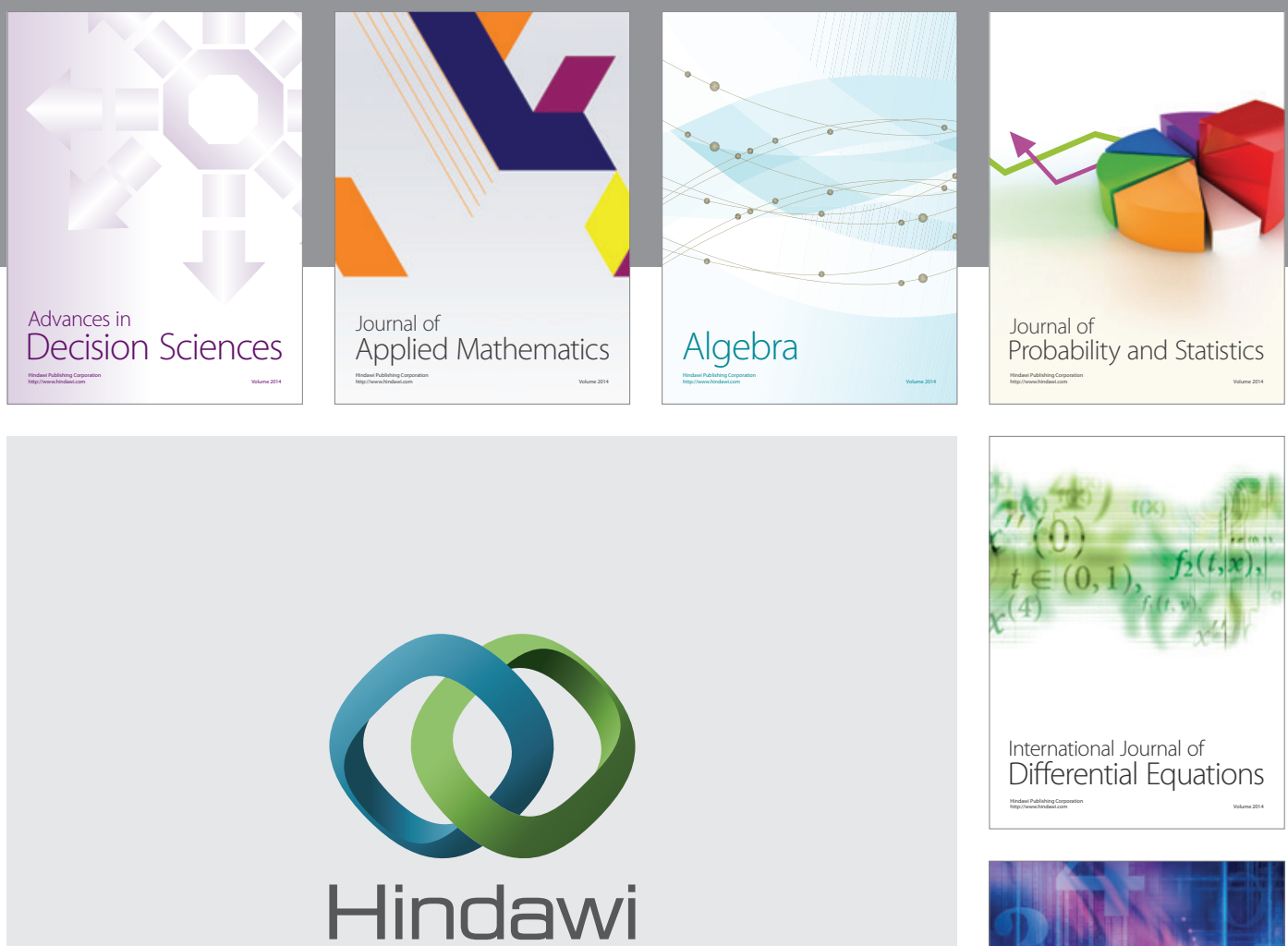

Submit your manuscripts at http://www.hindawi.com
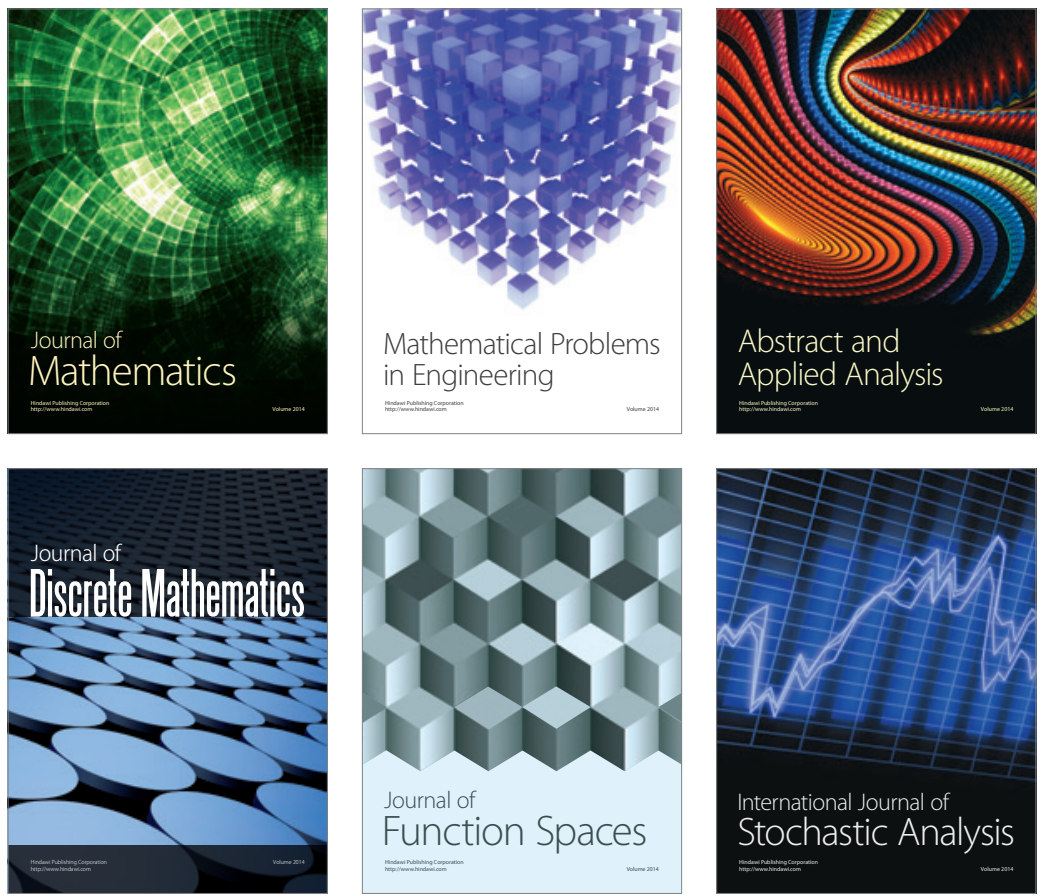

Journal of

Function Spaces

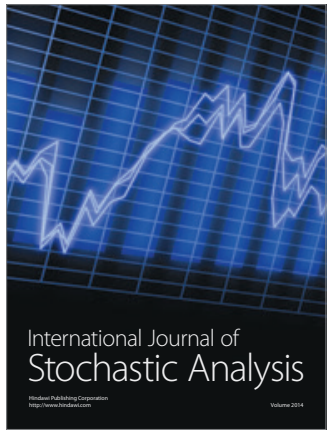

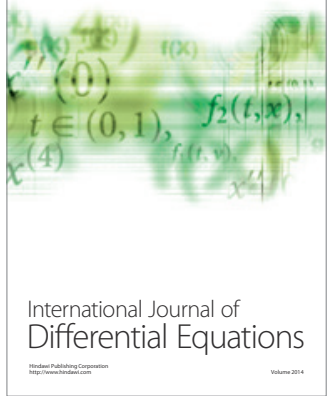
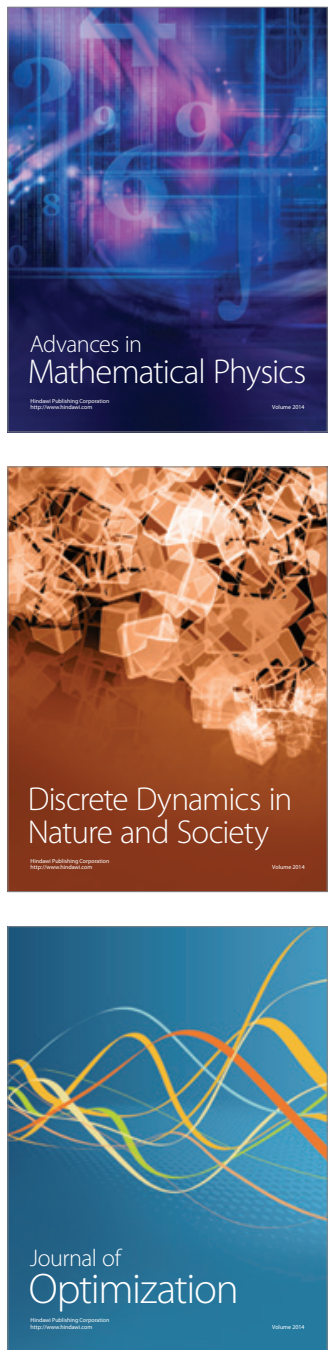